\title{
En direct du Congrès international d'aromathérapie et de phytothérapie appliquées Phyt'Arom (Grasse 2019)
}

\author{
P. Goetz \\ (C) Lavoisier SAS 2019
}

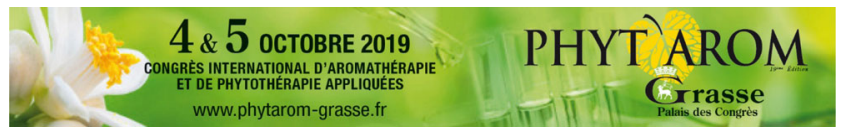

Le congrès Phyt'Arom est une ouverture indispensable sur une science et un art en pleine progression avec de plus en plus d'applications arrivant aujourd'hui à un enseignement en faculté de médecine.

Ce congrès est une scène de présentation de la médecine utilisant la phytoaromathérapie où doivent s'exposer les données les plus récentes dans ce domaine au niveau préclinique et clinique, sans oublier la galénique et l'usage traditionnel. Cette discipline est une thérapie dite alternative qui connaît de plus en plus de succès thérapeutique. Partie de la médecine traditionnelle, elle a de plus en plus de succès en médecine fondée sur les expérimentations et s'impose comme une alternative à une médecine de ville. Elle a de plus en plus de crédit auprès des patients si bien que les acteurs de la médecine doivent en acquérir la connaissance.

Ses perspectives peuvent se fonder sur les expérimentations animales mais aussi humaines. Les plantes et huiles essentielles médicinales se distinguent des compléments alimentaires, qui affluent par la méthodologie qui cerne bien ses effets cliniques, sa pharmacologie et des données toxicologiques.

Ce congrès s'adresse à l'ensemble des praticiens de santé : médecins, praticiens de thérapie alternative, infirmiers, kinésithérapie et masseurs, pharmaciens d'officine mais aussi ceux actifs au sein de laboratoire(s), petits et grands laboratoires de phytomédicament ou de compléments alimentaires, voire les spécialistes de dermocosmétologie.

Le congrès peut être un élément événementiel d'un DPC pour médecins déjà existants.

La ville de Grasse est connue pour son apport à l'industrie du parfum, mais aussi par sa situation dans une région de culture de plantes à huiles essentielles mais aussi d'autres

\section{P. Goetz $(\bowtie)$}

DU de phytothérapie, Paris-XIII, F-93017 Bobigny, France

e-mail : paul.goetz@wanadoo.fr plantes médicinales, soutenue activement par son conseil municipal. Le congrès bénéficie du soutien des industriels et des exposants qui nous permettent d'avoir accès aux formes galéniques. Cette manifestation est organisée par la ville de Grasse avec la coopération de membres actifs de la phytoaromathérapie (Copil) : pharmaciens, pharmacognostes, médecins phytoaromathérapeutes, infirmières, industriels, qui apportent la garantie d'interventions réalistes et actualisées en ce domaine.

C'est devant une salle comble que le maire de Grasse puis le Pr Anton ont ouvert le congrès de Phyt'arom 2019 de Grasse.

Cette année, le sujet principal tournait autour de l'aromathérapie qui occupe une place plus grande dans les EHPAD et entre dans l'activité hospitalière. L'utilisation en milieu hospitalier demande des protocoles bien réfléchis, et Évelyne Malaquin, auteur d'un « guide des HE », a tracé le cadre qui doit être adopté en aromathérapie scientifique et en milieu de soins : préconisations pour la pratique clinique, l'enseignement et la recherche, défis et perspectives, un an après.

Les Drs Tugba (lavande anesthésie) et Karadag ont montré l'utilisation de la lavande dans le cadre des troubles du sommeil.

M. Godet-Cicard a exposé les soins de bouche et sprays contre les mycoses cutanées et buccales en EHPAD.

J.-P. Giroux, président de Cosmed, est intervenu sur l'action endocrinienne des huiles essentielles et, en particulier, sur le risque des huiles essentielles susceptibles d'être des perturbateurs endocriniens.

Pour rappel, l'OMS définit les perturbateurs endocriniens (PE) tout substance « qui, seule ou en mélange, modifie les fonctions du système endocrinien et provoque des effets néfastes sur la santé chez des sujets sains sur leur descendance». Les hormones sont en perpétuelle modification pour permettre une adaptation physiologique aux contraintes environnementales. Cependant, les PE ont une action qui dépasse une simple adaptation physiologique, ils déclenchent un effet délétère sur l'organisme. Depuis 2018 et les dernières définitions des PE apportées par l'Europe, l'évaluation des PE est devenue obligatoire selon les réglementations REACH, 
pesticides et cosmétiques. Les personnes à risque face aux $\mathrm{PE}$ sont principalement les femmes enceintes (avortement spontané, souffrance fœtale, atrophie...) et les enfants à naître (cancer, stérilité, diabète...), d'où l'intérêt d'un modèle in vitro sur des cellules placentaires humaines. Un premier modèle développé au sein du laboratoire de toxicologie (Paris Descartes) a remporté de nombreux prix.

Y. Fillatre a présenté les résultats de la recherche de pesticides dans les huiles essentielles : analyse et réglementation.

L'intervention du Pr J. Kopferschmitt portait sur « Fake news, Infox » : informations fallacieuses en aromathérapie : un danger potentiel ? Quelle stratégie ?

M. Schwebel, lauréate 2018 du prix d'Aromathérapie clinique naturactive, a intitulé son intervention «Coup de pouce » : de l'apport d'une aromathérapie en gel sur la douleur chez les patients atteints de ténosynovite de De Quervain. Étude monocentrique, contrôlée, randomisée, en groupes parallèles, conduite en simple insu.

Anne Moro, $1^{\text {er }}$ prix 2018 de la fondation Gattefossé, a tenu à montrer l'utilisation des huiles essentielles lors de la chirurgie des fentes labiopalatines en mission humanitaire à Madagascar.

De nombreux ateliers portant sur des sujets de pratique médicale ont été suivis avec succès : Aromathérapie clinique $\&$ prise en charge globale du patient âgé (Isabelle El Khiari), Aromathérapie en maternité (Cécilia Trucas), La neuroimmunorhumatologie en pratique et les plantes dans les pathologies à autoanticorps (Dr Paul Goetz), Huiles essentielles et traumatologie (C. Busser), Accompagner les émotions : quelle place pour l'aromathérapie en psychiatrie (C. Maranzana), Apport de la phytoaromathérapie dans l'art dentaire, la maladie parodontale chronique et péri-implantite (F. Boukhobza), L'aromathérapie et la personne vieillissante (M. Godet-Cicard), Soins aromatiques par excellence sur les plaies infectées et très douloureuses (M. Kerkhof), Les potions végétales Santé \& Bien-être (M. Isted).

Cette agréable rencontre professionnelle s'est achevée sur l'enthousiasme des participants qui ont remercié l'excellente organisation du palais des Congrès et de L. Meineri et attendent avec impatience une prochaine édition. 